\title{
Conical versus gaussian profiled corrugated horn antennas
}

\author{
J. Teniente-Vallinas, R. Gonzalo-García, C. del-Río-Bocio* \\ Dept. of Electric and Electronic Engineering. Public University of Navarra \\ Campus de Arrosadía, s/n. 31006 Pamplona, (Navarra), Spain \\ Fax number: +34948 169720 Tfne. number: +34948169326 \\ e-mail: jorge.teniente@unavarra.es ramon@unavarra.es, carlos@unavarra.es
}

\begin{abstract}
In this paper, a study of two equal size corrugated horn antennas; a conical and a gaussian profiled horn antenna (GPHA) has been developed.

It will be demonstrated that GPHA's provide lower sidelobes and wider bandwidths, while conical corrugated horn antennas offer higher directivities for the same aperture. Both type of antennas provide low crosspolar levels.
\end{abstract}

\section{Introduction}

According to reference [1], corrugated horn antennas are supposed to provide a nearly perfect $\mathrm{HE}_{11}$ hybrid mode at its aperture while the contribution of any other hybrid modes as $\mathrm{HE}_{1 \mathrm{n}}$ and $\mathrm{EH}_{1 \mathrm{n}}$ (with $\mathrm{n} \geq 2$ ) should be reduced in order to obtain a low sidelobes and crosspolar levels.

It is known that the addition of $\mathrm{EH}_{1 \mathrm{n}}$ modes increases the crosspolar level of a corrugated horn antenna. Nevertheless, this paper shows that the idea of avoiding $\mathrm{HE}_{1 \mathrm{n}}$ modes to reduce sidelobes is not true. In fact, it will be proved that GPHA's provide a $\mathrm{HE}_{1 \mathrm{n}}$ mode mixture at the aperture as function of the relation between aperture radius $(R)$ and beamwaist radius $\left(\omega_{0}\right)$ of the fundamental gaussian beam to be generated (fig. 1).
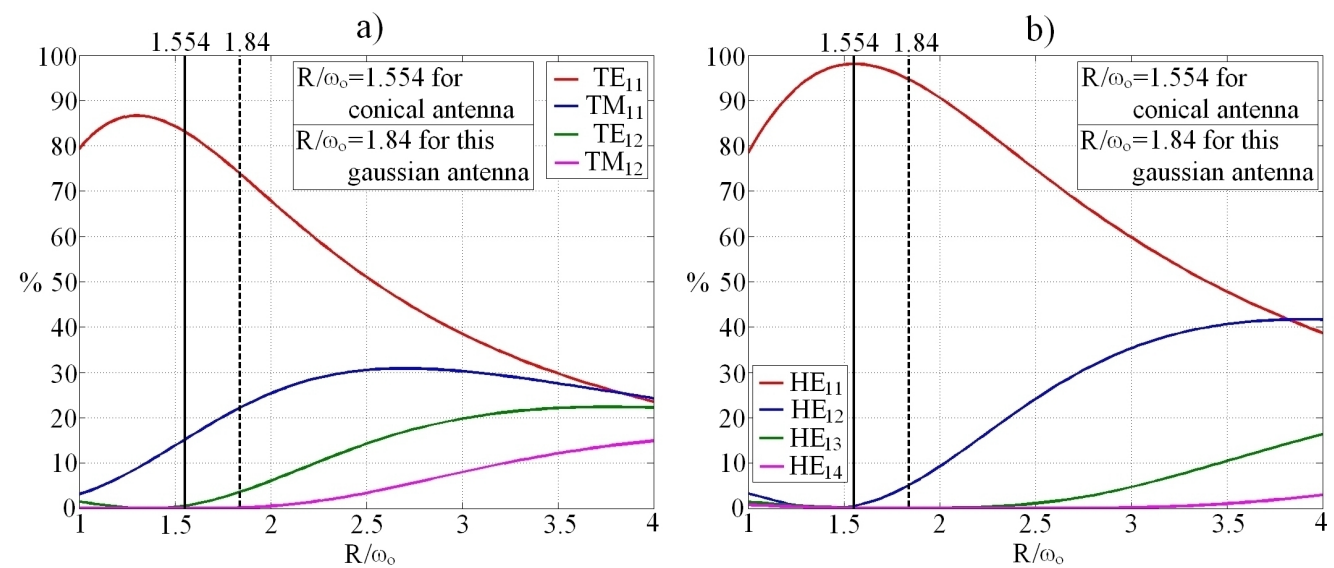

Fig. 1.- a) Fundamental gaussian mode decomposition in terms of TE and TM

b) Fundamental gaussian mode decomposition in terms of $\mathrm{HE}$ and $\mathrm{EH}$

\section{Relation between gaussian modes and waveguide modes}

The TE and TM basis define the modes inside of a smooth waveguide. On the other hand, the eigenmodes of a corrugated waveguide can be defined either as a mixture of TE and TM modes or by the HE and EH basis. This means that any HE or EH mode can be expanded as a combination of TE and TM modes (see table 1). 


\begin{tabular}{|c|c|c|c|c|c|c|}
\hline & $\mathrm{TE}_{11}(\%)$ & $\mathrm{TM}_{11}(\%)$ & $\mathrm{TE}_{12}(\%)$ & $\mathrm{TM}_{12}(\%)$ & $\mathrm{TE}_{13}(\%)$ & $\mathrm{TM}_{13}(\%)$ \\
\hline $\mathrm{HE}_{11}$ & 84.496 & 14.606 & 0.082 & 0.613 & 0.0036 & 0.121 \\
\hline
\end{tabular}

Table $1 . \mathrm{HE}_{11}$ decomposition in $\mathrm{TE}_{1 \mathrm{n}}$ and $\mathrm{TM}_{1 \mathrm{n}}$ smooth waveguide modes

Using this property, up to now the mode mixture of $85 \% \mathrm{TE}_{11}$ and $15 \% \mathrm{TM}_{11}$ has been used to provide a $\mathrm{HE}_{11}$ mode (see table 1). Furthermore, corrugated horn antennas have been always designed to provide at its output the aforementioned $\mathrm{TE}_{11^{-}}$ $\mathrm{TM}_{11}$ mixture.
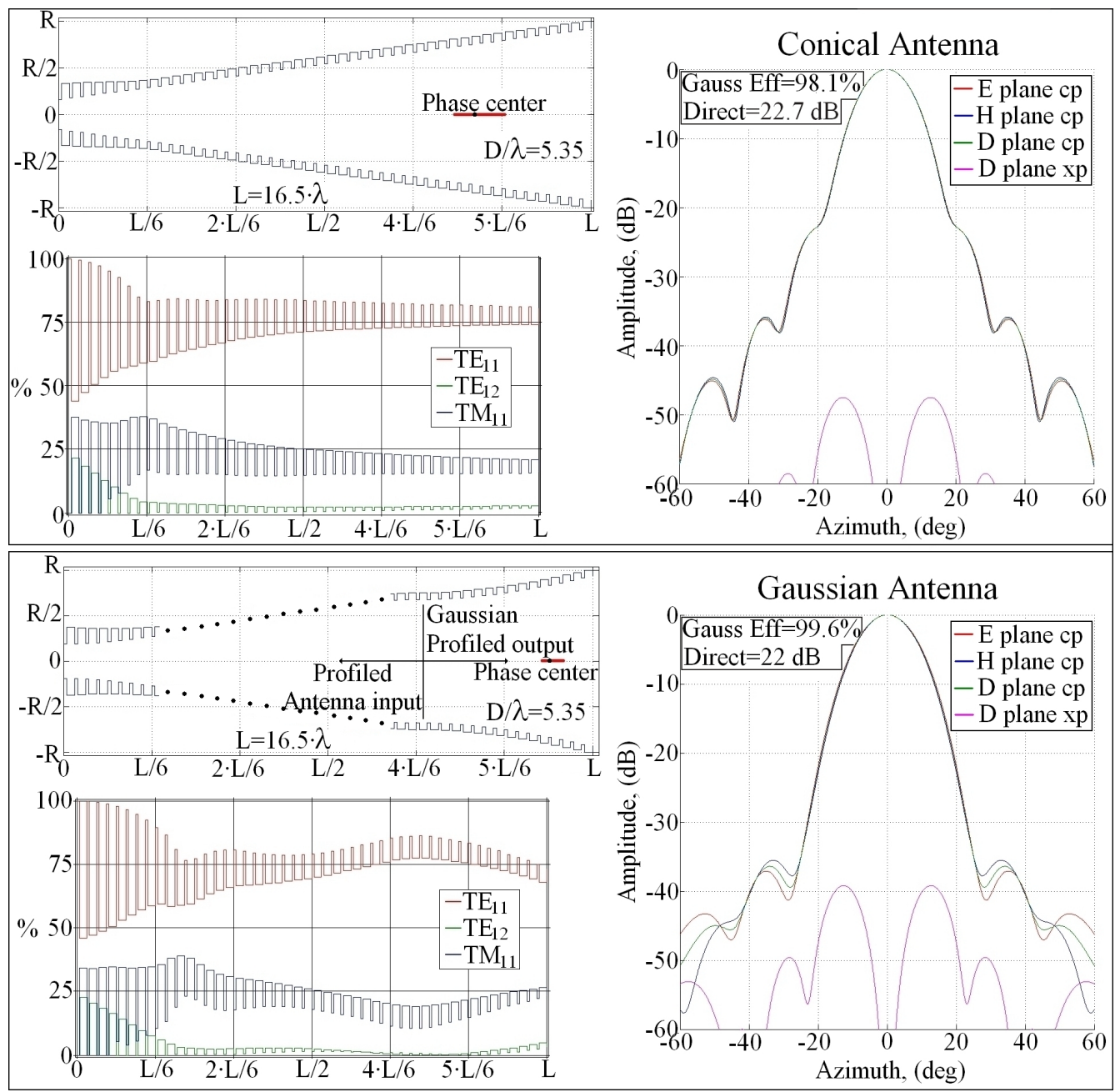

Fig. 2.- a) Conical and b) GPHA horn profiles together with the TE-TM mode evolution inside the antenna and the final radiation patterns at $\mathrm{f}_{0}$

When we started the development of GPHA's 6 years ago, the main idea was to generate a pure fundamental gaussian beam mode (which is a solution of paraxial free space) instead of an $\mathrm{HE}_{11}$ mode.

At this point, it should be noted that $\mathrm{HE}_{11}$ mode has been always known as a gaussian-like mode because its field is nearly a pure gaussian, in fact it is up to $98.1 \%$ efficient with a fundamental gaussian beam of $\mathrm{R} / \omega_{0}=1.554\left(\omega_{0} / \mathrm{R}=0.6435\right)$ (fig. 1). Nevertheless, there are some important differences between both cases. These aspects will be commented in the following section. 


\section{Conical Corrugated Horns versus Gaussian Profiled Corrugated Horns}

A conical corrugated horn antenna produces a field at its aperture that is nearly a pure $\mathrm{HE}_{11}$ mode with $\mathrm{HE}_{1 \mathrm{n}}$ modes lower than $-20 \mathrm{~dB}$ with respect to the fundamental mode, (fig. $3 \mathrm{~b}$ and fig. 1).

Looking at figure $3 \mathrm{a}$, one can see that for a rather good performance antenna, the pure $\mathrm{HE}_{11}$ has a restriction: it is impossible to obtain an antenna pattern with sidelobes lower than $-30 \mathrm{~dB}$ with directivities above $17 \mathrm{~dB}$.

However, GPHA's in a natural way produce a mixture of $\mathrm{HE}_{1 \mathrm{n}}$ modes corresponding with the figure $1 \mathrm{~b}$, for a given $\mathrm{R}$ and $\omega_{0}$. In figure $3 \mathrm{~b}$ significant reduction of sidelobe level is observed for GPHA's in relation with the equal-size conical counterpart.

It should be noted that both types of antennas present low amplitudes of crosspolarized $\mathrm{EH}_{1 \mathrm{n}}$ modes, but the GPHA is less frequency sensitive.

a)

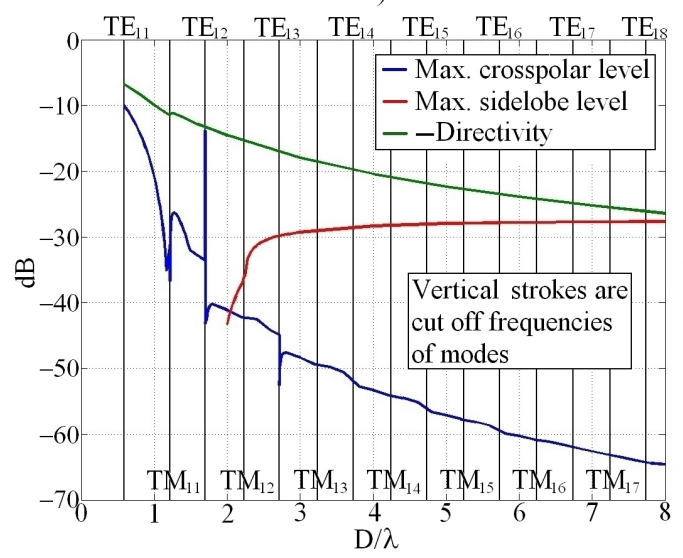

b)

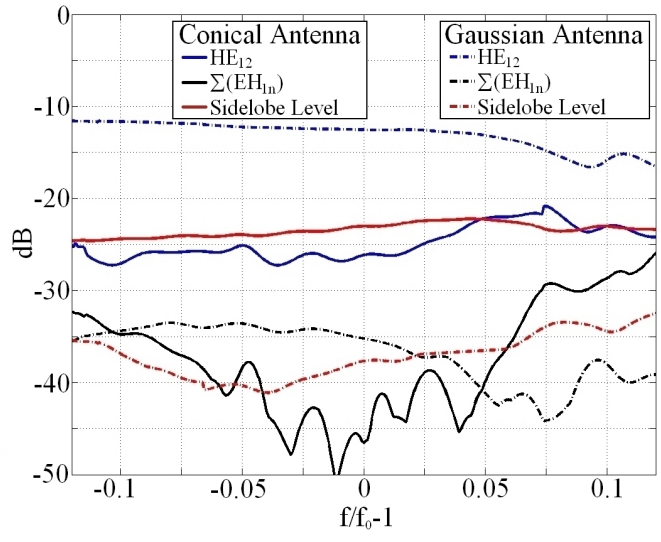

Fig. 3.- a) $\mathrm{HE}_{11}$ hybrid mode radiation properties against aperture diameter (D)

b) Levels of higher order $\mathrm{HE}_{1 \mathrm{n}}-\mathrm{EH}_{1 \mathrm{n}}$ modes at aperture and sidelobe level for the conical and the gaussian antennas against normalized frequency

The sidelobe level reduction for GPHA's can be evaluated looking at the aperture electric field of a conical corrugated antenna and a GPHA, (fig. 4a). The conical antenna provides a nearly perfect $\mathrm{HE}_{11}$ mode while the GPHA provides a narrower ilumination. As sidelobe level is directly related to the electric field on the aperture edges, the $\mathrm{HE}_{11}$ mode will always present a higher sidelobe level.

Furthermore, the fact of increasing the GPHA length will reduce even more the sidelobe level, while in the conical one, the sidelobe level keeps constant, since the aperture field distribution remains the same (fig. 2a) [2].

Another advantage of GPHA's is related to the bandwidth, as it can be seen from the stability of the curves in figure $3 b$ and $4 b$ [3].

However, the horn aperture ilumination efficiency is lower in a GPHA than in a conical horn (fig. 4a).

The efficency to a fundamental gaussian beam larger than $99.1 \%$ versus $98.2 \%$ over a $25 \%$ bandwidth and phase center position with nearly flat position for $15 \%$ bandwidth are improvements added by the use of a GPHA (fig. 4b). 

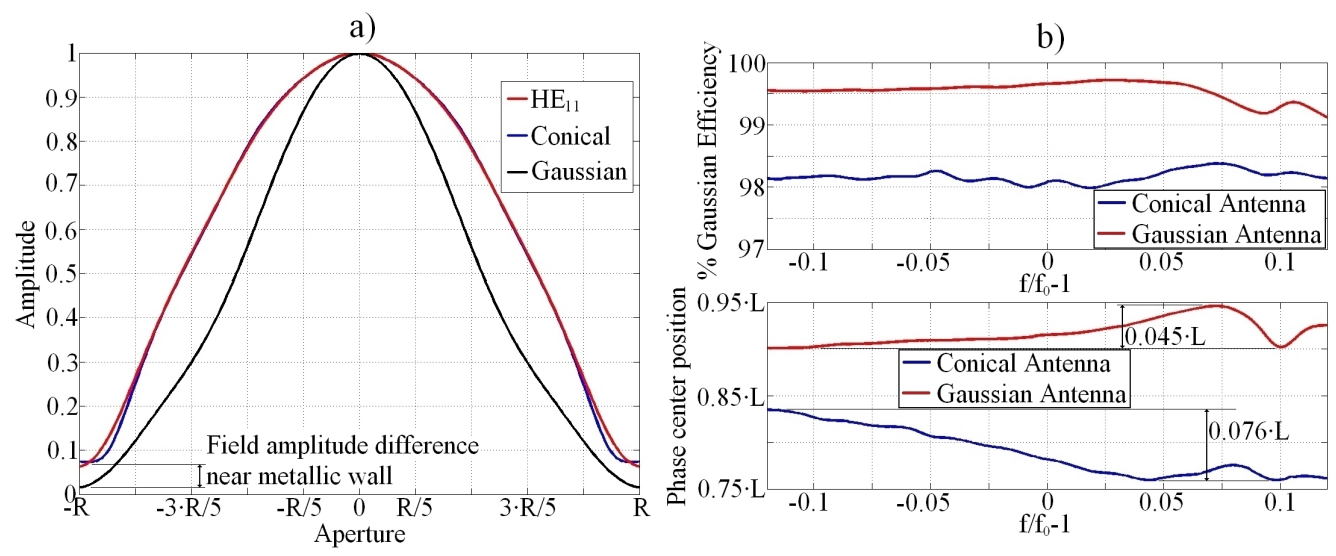

Fig. 4.- a) Aperture field of a conical corrugated horn antenna, a GPHA and a pure $\mathrm{HE}_{11}$ mode

b) Efficiency to fundamental gaussian beam and phase center position for a GPHA and a conical corrugated horn antenna

It should be pointed out that the phase center stability is a great advantage for ilumination of reflectors.

In general, for a given requirements, GPHA's offers a shorter solution than conical antennas.

\section{Conclusions}

Gaussian profiled corrugated horn antennas offer one of the best solutions for high performance horns where low sidelobes, wide bandwidth, low crosspolar levels, taper and phase center stability are important design parameters.

In spite of conical corrugated horn antennas illuminate more efficiently the antenna aperture than GPHA's, are unable to offer sidelobes lower than $-30 \mathrm{~dB}$. This lower aperture ilumination efficiency for the GPHA's doesn't lead to longer profiles; on the contrary, very often a shorter profile can be designed due to the better radiation features.

Nearly all of the systems that use a conical corrugated horn profile could be improved by the use of a GPHA.

\section{References}

[1] A. D. Olver, P. J. B. Clarricoats, A. A. Kishk and L. Shafai, "Microwave Horns and Feeds", IEE Electromagnetic Waves Series, Volume 39, 1994. ISBN America: 078031115 9. ISBN elsewhere: 0852968094

[2] C. del Río, R. Gonzalo and M. Sorolla, "High Purity Gaussian Beam Excitation by Optimal Horn Antenna", Procceedings of ISAP'96. Chiba, Japan.

[3] J. Teniente, R. Gonzalo and C. del Río, "Ultra-Wide Band Corrugated Gaussian Profiled Horn Antenna Design", 2001 IEEE AP-S International Symposium and USNC/URSI National Radio Science Meeting, 8-13 of July, Boston, Massachusetts, United States. 